Brief paper

\title{
Dynamic output feedback stabilization of switched linear systems with delay via a trajectory based approach
}

\author{
Saeed Ahmed ${ }^{\mathrm{a}, *}$, Frédéric Mazenc ${ }^{\mathrm{b}}$, Hitay Özbay ${ }^{\mathrm{a}}$ \\ a Department of Electrical and Electronics Engineering, Bilkent University, Ankara 06800, Turkey \\ ${ }^{\mathrm{b}}$ Inria, Laboratoire des Signaux et Systèmes (L2S, UMR CNRS 8506), CNRS, CentraleSupélec, Université Paris-Sud, 3 rue Joliot Curie, 91192, Gif-sur-Yvette, \\ France
}

\section{A R T I C L E I N F O}

\section{Article history:}

Received 26 January 2017

Received in revised form 19 December 2017

Accepted 2 February 2018

Available online 28 March 2018

\section{Keywords:}

Switched systems

Delay

Output feedback

Observer

Stabilization

\begin{abstract}
A B S T R A C T
A new technique is proposed to construct observers and to achieve output feedback stabilization of a class of continuous-time switched linear systems with a time-varying delay in the output. The delay is a piecewise continuous bounded function of time and no constraint is imposed on the delay derivative. For stability analysis, an extension of a recent trajectory based approach is used; this is fundamentally different from classical Lyapunov function based methods. A stability condition is given in terms of the upper bound on the time-varying delay to ensure global uniform exponential stability of the switched feedback system. The main result applies in cases where some of the subsystems of the switched system are not stabilizable and not detectable.
\end{abstract}

(c) 2018 Elsevier Ltd. All rights reserved.

\section{Introduction}

Switched systems have extensive applications in networks, automotive control, power systems, aircraft and air traffic control, process control, mechanical systems, and many other domains; see Lin and Antsaklis (2009), and the references therein. Due to this strong motivation, many questions related to switched systems such as stability (Liberzon, 2003; Liberzon \& Morse, 1999; Sun \& Ge, 2011), controllability (Liu, Lin, \& Chen, 2013; Sun, Ge, \& Lee, 2002), observability and reachability (Hespanha, Liberzon, Angeli, \& Sontag, 2005; Ji, Feng, \& Guo, 2007; Sun et al., 2002; Tanwani, Shim, \& Liberzon, 2013), and synthesis (Pettersson, 2003; Sun \& $\mathrm{Ge}, 2005)$, have been extensively studied in various contributions. Stability and stabilization are challenging problems pertaining to switched systems due to their hybrid nature and they are the main topic of the present paper.

There are mainly two approaches used in the literature for establishing the stability of switched systems:

\footnotetext{
This work is supported by the PHC Bosphore 2016 France-Turkey under Project No. 35634QM and the Scientific and Technological Research Council of Turkey (TÜBITAK) under Project No. EEEAG-115E820. The material in this paper was partially presented at the 2017 American Control Conference, May 24-26, 2017, Seattle, WA, USA. This paper was recommended for publication in revised form by Associate Editor Jamal Daafouz under the direction of Editor Richard Middleton.

* Corresponding author.

E-mail addresses: saeed.ahmed@bilkent.edu.tr (S. Ahmed), frederic.mazenc@12s.centralesupelec.fr (F. Mazenc), hitay@bilkent.edu.tr (H. Özbay).
}

(i) It is shown in Liberzon and Morse (1999) that existence of a common strict Lyapunov function is a necessary and sufficient condition for the switched system to be stable under arbitrary switching. On the other hand, when such a Lyapunov function exists, finding it may be a difficult task because it is an NP-hard problem; see Blondel and Tsitsiklis (1997). (ii) Liberzon and Morse (1999) also showed that even if a switched system does not possess a common strict Lyapunov function, it may be stable under a dwelltime requirement, typically derived using multiple strict Lyapunov functions. It is worth mentioning that multiple Lyapunov functions may lead to an undesirable attenuation property which can only be mitigated by imposing some strong assumptions; see Zhai, Hu, Yasuda, and Michel (2001).

Both of the above mentioned approaches are mainly developed for non-delayed systems. But measurement delays are present in many practical applications, such as chemical processes, aerodynamics and communication networks, and they are time-varying (see for instance Wu and Grigoriadis (2001) and Yan and Özbay (2005)). Therefore, the problem of stabilizing switched systems when a time-varying delay is present in the output is strongly motivated. State feedback stabilization of delayed switched linear systems is proposed in $\mathrm{Vu}$ and Morgansen (2010) using a combination of the multiple Lyapunov functions approach and the merging switching signal technique. An online and offline state feedback controller design for delayed switched linear systems in the detection of the switching signal are discussed in Xie and Wang (2005). Moreover, Koru, Delibaşı, and Özbay (2018) and Yan, Özbay, and Şansal (2014) present state 
feedback designs for delayed switched systems using a dwelltime based stability analysis approach. Note that Koru et al (2018), Vu and Morgansen (2010), Xie and Wang (2005) and Yan et al. (2014) assume that all of the subsystems of the switched system are controllable. Finally, a state feedback stabilization problem for a class of delayed switched systems is studied in Kim, Campbell, and Liu (2006) and Sun, Wang, Liu, and Zhao (2008) under the assumption that the subsystems satisfy a certain Hurwitz convex combination condition. A common Lyapunov function approach is used in Kim et al. (2006) and Sun et al. (2008) to carry out stability analysis.

Contributions of this study: We propose a new technique to design observers and stabilizing dynamic output feedbacks offering robust stability results with respect to the presence of a timevarying pointwise delay in the output of the switched linear system. To establish the stability of the closed-loop switched system, we develop an extension of the trajectory based stability result recently proposed in Mazenc and Malisoff (2015), and Mazenc, Malisoff, and Niculescu (2017). We wish to point out that the new extension of the trajectory based approach we state and prove in the present paper is of interest by itself: it can be applied to a wide range of systems, notably to families of systems with time-varying delays wider than those invoked in Mazenc and Malisoff (2015), and Mazenc, Malisoff et al. (2017), and therefore it is one of the important contributions of our work.

We think that our main result can be regarded as an extension of Kim et al. (2006), Koru et al. (2018), Sun et al. (2008), Vu and Morgansen (2010), Xie and Wang (2005), Yan et al. (2014) and Zhai, Hu, Yasuda, and Michel (2000), offering new advantages because, (i) our study does not assume that all the states are available for feedback, (ii) it is not limited to systems whose all subsystems are stabilizable and detectable, (iii) we use a new extension of trajectory based approach for stability analysis which circumvents the serious obstacle presented by the search for appropriate Lyapunov functions, (iv) the application of our results is not restricted to the class of delayed switched systems where all the convex combinations of the subsystems in the absence of control must be Hurwitz, (v) we allow the delay to be time-varying and piecewise continuous function of time, and we do not impose any constraint on the upper bound of the delay derivative.

Now, we point out that the present paper is a continuation of our conference paper (Mazenc, Ahmed, \& Özbay, 2017). We propose a significant extension of it by including, (i) dynamic output feedback stabilization, (ii) a new extension of trajectory based approach of Mazenc and Malisoff (2015) to produce less conservative results, (iii) a systematic way to compute an explicit value for the lower bound on the largest admissible delay for a broad family of switched systems so that when the delay is smaller than this bound, global uniform exponential stability (GUES) of the feedback switched systems is guaranteed. Moreover, we do not assume that the systems have synchronous switching sequences.

Organization of the paper: An extension of the trajectory based approach is given in Section 2. Section 3 is devoted to the main result of the paper. Section 4 discusses computational issues related to the delay bound. The results are illustrated by a numerical example in Section 5. Finally, we summarize and highlight our contributions in Section 6.

Notation: The notation will be simplified whenever no confusion can arise from the context. I denotes the identity matrix of any dimension. The usual Euclidean norm of vectors, and the induced norm of matrices, are denoted by $|\cdot|$. Given any constant $\tau>0$, we let $C\left([-\tau, 0], \mathbb{R}^{n}\right)$ denote the set of all continuous $\mathbb{R}^{n}$-valued functions that are defined on $[-\tau, 0]$. We abbreviate this set as $C_{\mathrm{in}}$, and call it the set of all initial functions. Also, for any continuous function $x:[-\tau, \infty) \rightarrow \mathbb{R}^{n}$ and all $t \geq 0$, we define $x_{t}$ by $x_{t}(\theta)=x(t+\theta)$ for all $\theta \in[-\tau, 0]$, i.e., $x_{t} \in C_{\text {in }}$ is the translation operator. A vector or a matrix is nonnegative (resp. positive) if all of its entries are nonnegative (resp. positive). We write $M \succ 0$ (resp. $M \preceq 0$ ) to indicate that $M$ is a symmetric positive definite (resp. negative semi-definite) matrix. For two vectors $V=\left(v_{1} \ldots v_{n}\right)^{\top}$ and $U=\left(u_{1} \ldots u_{n}\right)^{\top}$, we write $V \leq U$ to indicate that for all $i \in\{1, \ldots, n\}, v_{i} \leq u_{i}$.

\section{Extension of the trajectory based approach}

We now provide with an extension of the trajectory based approach given in Mazenc and Malisoff (2015).

Lemma 1. Let us consider a constant $T>0$ and l functions $z_{g}$ : $[-T,+\infty) \rightarrow[0,+\infty), g=1, \ldots$, l. Let $Z(t)=\left(z_{1}(t) \ldots z_{l}(t)\right)^{\top}$ and, for any $\theta \geq 0$ and $t \geq \theta$, define $\mathfrak{V}_{\theta}(t)=\left(\sup _{s \in[t-\theta, t]} z_{1}(s) \ldots\right.$ $\left.\sup _{s \in[t-\theta, t]} z_{l}(s)\right)^{\top}$. Let $\Upsilon \in \mathbb{R}^{l \times l}$ be a nonnegative Schur stable matrix. If for all $t \geq 0$, the inequalities $Z(t) \leq \Upsilon \mathfrak{V}_{T}(t)$ are satisfied, then $\lim _{t \rightarrow+\infty} z_{g}(t)=0 \quad \forall g=1, \ldots, l$.

Proof. Since $\Upsilon$ is Schur stable, there is an integer $q>1$ such that

$\left|\Upsilon^{q}\right| \sqrt{l}<1$

From Lemma 4 of Appendix A, we deduce that

$Z(t) \leq \Upsilon^{q} \mathfrak{V}_{q T}(t)$

for all $t \geq q T$. Consequently, $|Z(t)| \leq\left|\Upsilon^{q}\right|\left|\mathfrak{V}_{q T}(t)\right|$.

Using $\left|\mathfrak{V}_{q T}(t)\right| \leq \sqrt{l} \sup _{s \in[t-q T, t]}|Z(s)|$, we obtain

$|Z(t)| \leq\left|\Upsilon^{q}\right| \sqrt{l} \sup _{s \in[t-q T, t]}|Z(s)|$.

This inequality, in combination with the inequality (1) and Mazenc and Malisoff (2015, Lemma 1), allows us to conclude the result.

\section{Observer and control design}

We introduce a range dwell-time condition, i.e. a sequence of real numbers $t_{k}$ such that there are two positive constants $\underline{\delta}$ and $\bar{\delta}$ such that $t_{0}=0$ and for all $k \in \mathbb{Z}_{\geq 0}$,

$t_{k+1}-t_{k} \in[\underline{\delta}, \bar{\delta}]$.

Definition 1. Let $\pi=\left\{\left(i_{0}, t_{0}\right), \ldots,\left(i_{k}, t_{k}\right), \ldots, \mid i_{k} \in \Xi, k \in \mathbb{Z}_{\geq 0}\right\}$ be a switching sequence. The function $\sigma:[0, \infty) \rightarrow \Xi=$ $\{1, \ldots, n\}$ such that $\sigma(t)=i_{k}$ when $t \in\left[t_{k}, t_{k+1}\right)$ is called an associated switching signal.

We consider the continuous-time switched linear system:

$\left\{\begin{array}{l}\dot{x}(t)=A_{\sigma(t)} x(t)+B_{\sigma(t)} u(t) \\ y(t)=C_{\sigma(t)} x(t-\tau(t))\end{array}\right.$

with $x \in \mathbb{R}^{d_{x}}, u \in \mathbb{R}^{d_{u}}, y \in \mathbb{R}^{d_{y}}$, for all $t \geq 0, \tau(t) \in[0, \bar{\tau}]$ with $\bar{\tau}>0$ and an initial condition in $C_{\text {in }}$. The delay $\tau(t)$ is supposed to be a piecewise continuous function. For any $i \in \Xi, A_{i}, B_{i}$, and $C_{i}$ are real and constant matrices of compatible dimensions and $\sigma$ is a switching signal. We introduce an assumption which pertains to the stabilizability and the detectability of the system (4), but does not imply that all the pairs $\left(A_{i}, B_{i}\right)$ are stabilizable and all the pairs $\left(A_{i}, C_{i}\right)$ are detectable.

Assumption 1. There are matrices $K_{i}$ and $L_{i}$ for all $i \in \Xi$ and constants $T \geq \bar{\tau}, a \in[0,1), b \geq 0, c \in[0,1)$ and $d \geq 0$ such that the solutions of the system

$\dot{\alpha}(t)=M_{\sigma(t)} \alpha(t)+\zeta(t)$ 
with $M_{i}=A_{i}+B_{i} K_{i}$ and $\zeta$ being a piecewise continuous function, satisfy

$|\alpha(t)| \leq a|\alpha(t-T)|+b \sup _{\ell \in[t-T, t]}|\zeta(\ell)|$

for all $t \geq T$. Similarly, the solutions of the system

$\dot{\beta}(t)=N_{\sigma(t)} \beta(t)+\eta(t)$

with $N_{i}=A_{i}+L_{i} C_{i}$ and $\eta$ being a piecewise continuous function, satisfy the following inequality for all $t \geq T$

$|\beta(t)| \leq c|\beta(t-T)|+d \sup _{\ell \in[t-T, t]}|\eta(\ell)|$.

Theorem 1. Let the system (4) satisfy Assumption 1 and, $s_{1}, s_{2}$ and $s_{3}$ be defined by

$s_{1}=\sup _{i \in \Xi}\left|B_{i} K_{i}\right|, s_{2}=\sup _{i \in \Xi}\left|L_{i} C_{i}\right|, s_{3}=\sup _{i \in \Xi}\left|M_{i}\right|$.

If

$\tau(t) \leq \bar{\tau}<\bar{\tau}_{u}$

for all $t \geq 0$, where

$\bar{\tau}_{u}=\frac{(1-a)(1-c)}{d s_{1} s_{2}\left((1-a)+b s_{3}\right)}$,

then the origin of the following feedback system is GUES:

$\left\{\begin{aligned} \dot{x}(t)= & A_{\sigma(t)} x(t)+B_{\sigma(t)} K_{\sigma(t)} \hat{x}(t) \\ \dot{\hat{x}}(t)= & A_{\sigma(t)} \hat{x}(t)+B_{\sigma(t)} K_{\sigma(t)} \hat{x}(t) \\ & +L_{\sigma(t)}\left[C_{\sigma(t)} \hat{x}(t)-y(t)\right] .\end{aligned}\right.$

Proof. Let us introduce $\tilde{x}(t)=\hat{x}(t)-x(t)$. Then

$\dot{\tilde{x}}(t)=A_{\sigma(t)} \tilde{x}(t)+L_{\sigma(t)}\left[C_{\sigma(t)} \hat{x}(t)-C_{\sigma(t)} x(t-\tau(t))\right]$.

As an immediate consequence, using the definitions of the matrices $M_{i}$ and $N_{i}$, we obtain

$\left\{\begin{array}{l}\dot{x}(t)=M_{\sigma(t)} x(t)+B_{\sigma(t)} K_{\sigma(t)} \tilde{x}(t) \\ \dot{\tilde{x}}(t)=N_{\sigma(t)} \tilde{x}(t)+L_{\sigma(t)} C_{\sigma(t)}[x(t)-x(t-\tau(t))] .\end{array}\right.$

From Assumption 1 and the equality $x(\ell)-x(\ell-\tau(\ell))=\int_{\ell-\tau(\ell)}^{\ell}$ $\left[M_{\sigma(m)} x(m)+B_{\sigma(m)} K_{\sigma(m)} \tilde{x}(m)\right] d m$, it follows that, for all $t \geq T+\bar{\tau}$,

$|x(t)| \leq a|x(t-T)|+b \sup _{\ell \in[t-T, t]}\left|B_{\sigma(\ell)} K_{\sigma(\ell)} \tilde{x}(\ell)\right|$,

$|\tilde{x}(t)| \leq c|\tilde{x}(t-T)|+d \sup _{\ell \in[t-T, t]} \mid L_{\sigma(\ell)} C_{\sigma(\ell)}$

$\times \int_{\ell-\tau(\ell)}^{\ell}\left[M_{\sigma(m)} x(m)+B_{\sigma(m)} K_{\sigma(m)} \tilde{x}(m)\right] d m \mid$.

Using the constants defined in (9), we deduce from (13) and (14) that $(x(t), \tilde{x}(t))$ satisfies:

$$
\begin{aligned}
|x(t)| \leq & a|x(t-T)|+b s_{1} \sup _{\ell \in[t-T-\bar{\tau}, t]}|\tilde{x}(\ell)|, \\
|\tilde{x}(t)| \leq & d s_{2} s_{3} \bar{\tau} \sup _{\ell \in[t-T-\bar{\tau}, t]}|x(\ell)| \\
& +\left(c+d s_{1} s_{2} \bar{\tau}\right) \sup _{\ell \in[t-T-\bar{\tau}, t]}|\tilde{x}(\ell)| .
\end{aligned}
$$

Lemma 1 ensures that the origin of (12) is GUES if

$\left[\begin{array}{cc}a & b s_{1} \\ d s_{2} s_{3} \bar{\tau} & d s_{1} s_{2} \bar{\tau}+c\end{array}\right]$ is Schur stable, which is equivalent to

$$
\frac{\frac{a+c+d s_{1} s_{2} \bar{\tau}}{2}+}{\sqrt{\left(\frac{a+c+d s_{1} s_{2} \bar{\tau}}{2}\right)^{2}-a c-d s_{1} s_{2}\left(a-b s_{3}\right) \bar{\tau}}<1,}
$$

from which we derive the simpler condition (10).

\section{Parameters of the delay bound}

In this section, we illustrate a method to determine the constants $a, b, c$, and $d$ appearing in Assumption 1.

Consider a continuous-time switched linear system

$\dot{\xi}(t)=\Omega_{\sigma(t)} \xi(t)+\vartheta(t)$,

where $\xi \in \mathbb{R}^{d_{\xi}}$, the switching signal $\sigma$ is associated to a sequence $t_{k}$ of the type of those introduced in Section 3 and $\vartheta$ is a piecewise continuous function.

Lemma 2. Let the system (15) be such that there are real numbers $d_{1}>0, d_{2}>0, \mu \geq 1, \gamma>0$ and symmetric positive definite matrices $Q_{l}, l \in \Xi$, such that the LMIs

$$
\begin{aligned}
& d_{1} I \preceq Q_{i} \preceq d_{2} I, \\
& Q_{i} \preceq \mu Q_{j}, \\
& \Omega_{i}^{\top} Q_{i}+Q_{i} \Omega_{i} \preceq-\gamma Q_{i}
\end{aligned}
$$

are satisfied for all $i, j \in \Xi$. Moreover, the constant $\mu_{\triangle}=\mu e^{-\gamma \underline{\delta}} i s$ such that

$\mu_{\triangle}<1$.

Then, along the trajectory of (15), the inequality

$|\xi(t)| \leq \sqrt{\frac{d_{2}}{d_{1}} \mu \mu_{\triangle}^{\rho} e^{\gamma \bar{\delta}}}|\xi(t-T)|+\sqrt{\mu \frac{d_{2}}{\gamma d_{1}} T} \sup _{\ell \in[t-T, t]}|\vartheta(\ell)|$

holds for all $t \geq T$ where $T>0$ and $\rho$ is a positive integer depending on the choice of $T$ such that for all $t \in\left[t_{k}, t_{k+1}\right)$, we have $t-T \in\left[t_{k-\rho-1}, t_{k-\rho}\right)$. Moreover, we have $\sqrt{\frac{d_{2}}{d_{1}} \mu \mu_{\triangle}^{\rho} e^{\gamma \bar{\delta}}}<1$ when $\rho>\frac{1}{\ln \left(\mu_{\Delta}\right)}\left[\ln \left(\frac{d_{1}}{d_{2} \mu}\right)-\gamma \bar{\delta}\right]$.

For the proof of Lemma 2, see Appendix B.

Remark 1. 1. Note that (19) holds if and only if $\underline{\delta}>\frac{\ln (\mu)}{\gamma}$, which defines a minimum dwell-time condition.

2. Conditions of Lemma 2 are always satisfied when the matrices $\Omega_{i}, \forall i \in \Xi$, are Hurwitz; i.e., one can always find symmetric positive definite matrices $Q_{i}, i \in \Xi$, and real numbers $d_{1}>0$, $d_{2}>0, \mu \geq 1, \gamma>0$ satisfying the LMIs (16), (17), and (18). In the next section we illustrate an alternative approach for the case where some of $\Omega_{i}$ 's are not Hurwitz.

\section{Illustrative example}

Consider the continuous-time switched linear system (4) with $x \in \mathbb{R}^{2}, \tau \in[0, \bar{\tau})$,

$\sigma(t)= \begin{cases}1 & \text { if } 4 \ell \kappa \leq t<(4 \ell+3) \kappa \\ 2 & \text { if }(4 \ell+3) \kappa \leq t<4(\ell+1) \kappa,\end{cases}$

where $\kappa>0$ is to be determined, $\ell=0,1,2, \ldots$, and

$A_{1}=\left[\begin{array}{cc}0 & -1 / 2 \\ 2 / 5 & 0\end{array}\right], B_{1}=\left[\begin{array}{ll}0 & 0 \\ 0 & 0\end{array}\right], C_{1}=\left[\begin{array}{ll}1 & 0 \\ 0 & 1\end{array}\right]$, 
$A_{2}=\left[\begin{array}{cc}0 & -2 / 5 \\ 1 / 2 & 0\end{array}\right], B_{2}=\left[\begin{array}{ll}1 & 0 \\ 0 & 1\end{array}\right], C_{2}=\left[\begin{array}{ll}0 & 0 \\ 0 & 0\end{array}\right]$.

Let us observe that the subsystem $\left(A_{1}, B_{1}, C_{1}\right)$ is not stabilizable but it is detectable whereas the subsystem $\left(A_{2}, B_{2}, C_{2}\right)$ is stabilizable but not detectable. Moreover, in the absence of control, no convex combination of the $A_{1}$ and $A_{2}$ is Hurwitz. Furthermore, the subsystems cannot be stabilized by a static output feedback $u=K_{i} y$. In this example, we have $\underline{\delta}=\kappa$ and $\bar{\delta}=3 \kappa$ and the switchings are periodic with a period of $4 \kappa$. We will determine a set of parameters for the delay bound depending on $\kappa$.

\subsection{Preliminary result}

First, we provide a preliminary result which shows how Assumption 1 can be satisfied in this particular example where some of the subsystems of the switched systems are not stabilizable and not detectable.

Lemma 3. Consider the switched linear system

$\dot{z}(t)=\Gamma_{\sigma(t)} z(t)+\varrho(t)$

with $\sigma$ defined by (20), and let $\Gamma_{1} \in \mathbb{R}^{2 \times 2}, \Gamma_{2} \in \mathbb{R}^{2 \times 2}$ and $\kappa>0$ be such that the matrix $S_{\kappa}:=e^{\Gamma_{2} \kappa} e^{3 \Gamma_{1} \kappa}$ is Schur stable. Let $\Phi_{\star}$ be the state transition matrix of the system (21) with $\varrho=0$ :

$\frac{\partial \Phi_{\star}}{\partial t}(t, s)=\Gamma_{\sigma(t)} \Phi_{\star}(t, s), \Phi_{\star}(s, s)=I$,

for all $t \in \mathbb{R}$ and $s \in \mathbb{R}$. Then, for all $s \geq 0, t \geq s$

$\left|\Phi_{\star}(t, s)\right| \leq p_{1} e^{-p_{2}(t-s)}$

with $p_{1}=e^{8 \kappa \max \left\{\left|\Gamma_{1}\right|,\left|\Gamma_{2}\right|\right\}} c_{\kappa} e^{2 d_{\kappa}}$ and $p_{2}=d_{\kappa} / 4 \kappa$, where $c_{\kappa}>1$ and $d_{\kappa}>0$ are such that for all $m \in \mathbb{N}$,

$\left|S_{\kappa}^{m}\right| \leq c_{\kappa} e^{-d_{\kappa} m}$.

Moreover, for all $T>0$,

$$
\begin{aligned}
|z(t)| \leq & p_{1} e^{-p_{2} T}|z(t-T)| \\
& +\frac{p_{1}\left(1-e^{-p_{2} T}\right)}{p_{2}} \sup _{\ell \in[t-T, t]}|\varrho(\ell)| .
\end{aligned}
$$

For the proof of Lemma 3, see Appendix C.

Remark 2. Since $p_{2}>0$, then $p_{1} e^{-p_{2} T}<1$ when $T>\frac{\ln \left(p_{1}\right)}{p_{2}}$, which determines a lower bound for $T$.

\subsection{Output feedback stabilization}

Let us choose the gain matrices as

$K_{2}=\left[\begin{array}{cc}-1 / 2 & 0 \\ 0 & -4 / 7\end{array}\right], L_{1}=\left[\begin{array}{cc}-3 / 5 & 0 \\ 0 & -4 / 5\end{array}\right]$.

Setting $\Gamma_{1}=M_{1}=A_{1}$ and $\Gamma_{2}=M_{2}=A_{2}+B_{2} K_{2}$, one can easily corroborate that (23) is satisfied with the choice of $\kappa=0.1$, $c_{k}=1.01$, and $d_{k}=0.001$ for all $m \in \mathbb{N}$. Setting $z=\alpha$, $\Omega_{i}=\Gamma_{i}=M_{i}$ for $i \in\{1,2\}$, and $\varrho=\zeta$, it can be easily verified that (22) is satisfied by (5) with $p_{1}=e^{8 \kappa \max \left\{\left|\Gamma_{1}\right|,\left|\Gamma_{2}\right|\right\}} c_{\kappa} e^{2 d_{\kappa}}=1.7142$ and $p_{2}=d_{\kappa} / 4 \kappa=1.0025$. Using Lemma 3 with $T=6$, one can observe that the solutions of system (5) satisfy (6) with $a=$ $p_{1} e^{-p_{2} T}=0.0042, b=\left(p_{1} / p_{2}\right)\left(1-e^{-p_{2} T}\right)=1.7057$. A similar analysis shows that the solutions of system (7) satisfy (8) with $c=$ $0.0052, d=2.1156$ and $T=6$. Therefore, we conclude that the switched delay system satisfies Assumption 1. Finally, application of Theorem 1 with $s_{1}=0.5714, s_{2}=0.8, s_{3}=0.7611$, and with the preceding choices of the parameters yields $\bar{\tau}_{u}=0.4465$. Fig. 1

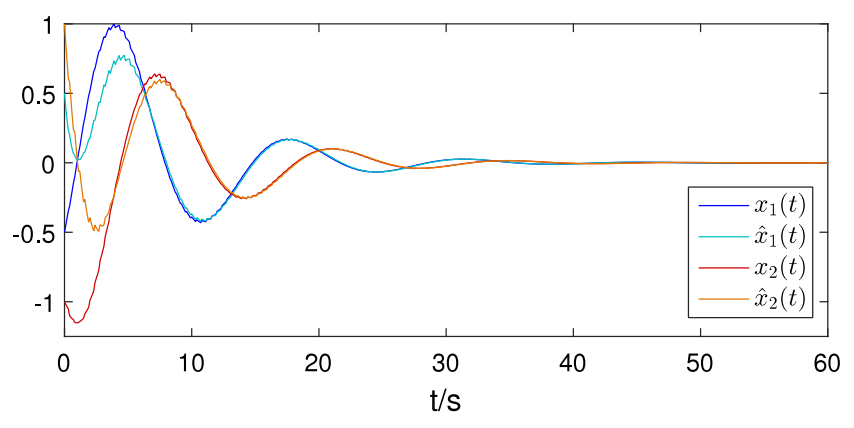

Fig. 1. Simulation results.

shows the simulation of system (12) for this particular example for a piecewise continuous sawtooth function $\tau(t)$ of a fundamental frequency of $1 \mathrm{~Hz}$ described by $\tau(t)=0.2(t-\lfloor t\rfloor)$ where the switching signal $\sigma(t)$ is given by (20) with $\kappa=0.1$. The initial conditions are chosen to be $x_{1}(0)=-0.5, x_{2}(0)=-1, \hat{x}_{1}(0)=0.5$, and $\hat{x}_{2}(0)=1$, and the sample rate is $1 \mathrm{kHz}$.

It is worth emphasizing here that Vu and Morgansen (2010) assumes that all of the modes of the delayed switched system are controllable and Sun et al. (2008) requires the derivative of the delay to be bounded which makes it impossible to apply their results to this example; and it also seems to us that there is no direct way to extend them to the output feedback case considered in this paper.

\section{Conclusions}

We presented dynamic output feedback stabilization results for systems with switches in the difficult case where a time-varying pointwise delay in the output is present. The technique of proof we proposed is based on the recent trajectory based approach. To solve the conservatism problem we encountered in Mazenc, Ahmed et al. (2017), we developed an extension of the main result of Mazenc and Malisoff (2015), which is of interest for its own sake. Many extensions of the results of the present paper are possible, pertaining for instance to design of $K_{i}$ and $L_{i}$ for maximization of the delay bound, robustness issues with respect to disturbances, the presence of a delay in the input, the design of reduced order observers and extensions to families of nonlinear systems.

\section{Appendix A. Technical lemma}

Lemma 4. Let $R \in \mathbb{R}^{m \times m}$ be a nonnegative matrix. Let us consider functions $w_{j}:[0,+\infty) \rightarrow[0,+\infty), j=1, \ldots, m$, and a constant $h>0$ such that for all $t \geq h, w=\left(w_{1} \ldots w_{m}\right)^{\top}$ satisfies

$w(t) \leq R \zeta(t)$

with $\zeta(t)=\left(\sup _{\ell \in[t-h, t]} w_{1}(\ell) \ldots \sup _{\ell \in[t-h, t]} w_{m}(\ell)\right)^{\top}$. Then, for all integer $k$ larger than 1 , and all $t \geq k h$, we have $w(t) \leq R^{k} \Psi_{k}(t)$ with $\Psi_{k}(t)=\left(\sup _{\ell \in[t-k h, t]} w_{1}(\ell) \ldots \sup _{\ell \in[t-k h, t]} w_{m}(\ell)\right)^{\top}$.

Proof. We prove the lemma by induction:

Induction Assumption: There is $l \in \mathbb{N}, l>0$ such that the result of Lemma 4 holds for all $k \in\{1, \ldots, l\}$.

Step 1: The assumption is satisfied at the step 1.

Step $l$ : Let us assume that it is satisfied at the step $l \geq 1$. Then the inequalities

$w(t) \leq R^{l} \Psi_{l}(t)$ 
hold for all $t \geq l h$. From (A.1), we deduce that for all $t \geq(l+1) h$ and $\ell \in[t-\overline{l h}, t]$, the inequalities

$\left(\begin{array}{c}w_{1}(\ell) \\ \vdots \\ w_{m}(\ell)\end{array}\right) \leq R\left(\begin{array}{c}\sup _{s \in[\ell-h, \ell]} w_{1}(s) \\ \vdots \\ \sup _{s \in[\ell-h, \ell]} w_{m}(s)\end{array}\right) \leq R \Psi_{l+1}(t)$

hold. It follows that

$\Psi_{l}(t) \leq R \Psi_{l+1}(t)$.

By combining (A.2) and (A.3), we deduce that

$w(t) \leq R^{l+1} \Psi_{l+1}(t)$

for all $t \geq(l+1) h$. Thus the induction assumption is satisfied at the step $\bar{l}+1$. This concludes the proof.

\section{Appendix B. Proof of Lemma 2}

Let us define Lyapunov functions:

$\mathcal{V}_{i}(\xi)=\xi^{\top} Q_{i} \xi, \forall i \in \Xi$.

We deduce from (18) that when $\sigma(t)=i$, then the derivative of $\mathcal{V}_{i}$ along the trajectories of (15) satisfies

$$
\begin{aligned}
\dot{\mathcal{V}}_{i}(\xi(t)) & \leq-2 \gamma \mathcal{V}_{i}(\xi(t))+2 \xi(t)^{\top} Q_{i} \vartheta(t) \\
& \leq-\gamma \mathcal{V}_{i}(\xi(t))+\frac{1}{\gamma} \vartheta(t)^{\top} Q_{i} \vartheta(t)
\end{aligned}
$$

where the last inequality is deduced from the Young's inequality. Now, let us integrate (B.1) between two instants $s$ and $t, t \geq s$, belonging to the same sampling interval where $\sigma(t)=l$. Then

$$
\begin{aligned}
\mathcal{V}_{l}(\xi(t)) \leq & e^{\gamma(s-t)} \mathcal{V}_{l}(\xi(s)) \\
& +\frac{1}{\gamma} \int_{s}^{t} e^{\gamma m-\gamma t} \vartheta(m)^{\top} Q_{l} \vartheta(m) d m \\
\leq & e^{\gamma(s-t)} \mathcal{V}_{l}(\xi(s))+\frac{d_{2}}{\gamma} \int_{s}^{t} e^{\gamma m-\gamma t}|\vartheta(m)|^{2} d m,
\end{aligned}
$$

where the last inequality is a consequence of (16). Now, let us consider $T>0, t \geq T$ such that $t \in\left[t_{k}, t_{k+1}\right)$ for some $k \in \mathbb{Z}_{\geq 0}$ and let $\rho \in \mathbb{N}$ be such that $t-T \in\left[t_{k-\rho-1}, t_{k-\rho}\right)$. From (B.2), we deduce that

$$
\begin{gathered}
\mathcal{V}_{\sigma\left(t_{k}\right)}(\xi(t)) \leq e^{-\gamma\left(t-t_{k}\right)} \mathcal{V}_{\sigma\left(t_{k}\right)}\left(\xi\left(t_{k}\right)\right) \\
\quad+\frac{d_{2}}{\gamma} \int_{t_{k}}^{t} e^{\gamma m-\gamma t}|\vartheta(m)|^{2} d m \\
\leq \mu e^{-\gamma\left(t-t_{k}\right)} \mathcal{V}_{\sigma\left(t_{k-1}\right)}\left(\xi\left(t_{k}\right)\right)+\frac{d_{2}}{\gamma} \int_{t_{k}}^{t}|\vartheta(m)|^{2} d m,
\end{gathered}
$$

where the last inequality is a consequence of (17). For similar reasons,

$$
\begin{aligned}
\mathcal{V}_{\sigma\left(t_{k-1}\right)}\left(\xi\left(t_{k}\right)\right) \leq & \mu_{\Delta} \mathcal{V}_{\sigma\left(t_{k-2}\right)}\left(\xi\left(t_{k-1}\right)\right) \\
& +\frac{d_{2}}{\gamma} \int_{t_{k-1}}^{t_{k}}|\vartheta(m)|^{2} d m \\
\vdots & \mu_{\triangle} \mathcal{V}_{\sigma\left(t_{k-\rho-1}\right)}\left(\xi\left(t_{k-\rho}\right)\right) \\
& +\frac{d_{2}}{\gamma} \int_{t_{k-\rho}}^{t_{k-\rho+1}}|\vartheta(m)|^{2} d m \\
\mathcal{V}_{\sigma\left(t_{k-\rho}\right)}\left(\xi\left(t_{k-\rho+1}\right)\right) \leq & e^{\gamma\left(t-T-t_{k-\rho}\right)} \mathcal{V}_{\sigma\left(t_{k-\rho-1}\right)}(\xi(t-T)) \\
\mathcal{V}_{\sigma\left(t_{k-\rho-1}\right)}\left(\xi\left(t_{k-\rho}\right)\right) \leq & +\frac{d_{2}}{\gamma} \int_{t-T}^{t_{k-\rho}}|\vartheta(m)|^{2} d m .
\end{aligned}
$$

Combining (B.3), (B.4) and (B.5), and then using the definition of range dwell-time condition from (3), we get

$$
\begin{aligned}
\mathcal{V}_{\sigma\left(t_{k}\right)}(\xi(t)) \leq & \mu \mu_{\triangle}^{\rho} e^{\gamma \bar{\delta}} \mathcal{V}_{\sigma\left(t_{k-\rho-1}\right)}(\xi(t-T)) \\
& +\mu \frac{d_{2}}{\gamma} \int_{t-T}^{t}|\vartheta(m)|^{2} d m
\end{aligned}
$$

Using (16) and the inequality $\sqrt{p_{1}+p_{2}} \leq \sqrt{p_{1}}+\sqrt{p_{2}}$ for all $p_{1} \geq 0$, $p_{2} \geq 0$, we obtain

$|\xi(t)| \leq \sqrt{\frac{d_{2}}{d_{1}} \mu \mu_{\triangle}^{\rho} e^{\gamma \bar{\delta}}}|\xi(t-T)|+\sqrt{\mu \frac{d_{2}}{\gamma d_{1}} T} \sup _{\ell \in[t-T, t]}|\vartheta(\ell)|$.

Since (19) holds and $T$ is arbitrarily large, one can choose $T$ such that the corresponding $\rho$ is so that $\sqrt{\frac{d_{2}}{d_{1}} \mu \mu_{\Delta}^{\rho} e^{\gamma \bar{\delta}}}<1$. This concludes the proof.

\section{Appendix C. Proof of Lemma 3}

Let us introduce a sequence: $g_{\ell}=4 \ell \kappa$. Then for all integer $n>0, z\left(g_{\ell}\right)=S_{\kappa}^{n} z\left(g_{\ell-n}\right)$. Thus $\Phi_{\star}\left(g_{\ell}, g_{\ell-n}\right)=S_{\kappa}^{n}$. Let $t \in \mathbb{R}$ and $s \in \mathbb{R}$ be such that $t>s \geq t-4 \kappa$. Then

$\left|\Phi_{\star}(t, s)\right| \leq e^{4 \kappa \max \left\{\left|\Gamma_{1}\right|,\left|\Gamma_{2}\right|\right\}}$.

Now, let $t \in \mathbb{R}$ and $s \in \mathbb{R}$ be such that $t+4 \kappa>s$. Then there is $\ell$ such that $t \in\left[g_{\ell}, g_{\ell+1}\right)$ and $r \in \mathbb{N}, r>0$ such that $s \in\left[g_{\ell-r-1}, g_{\ell-r}\right)$. Then

$\left|\Phi_{\star}(t, s)\right| \leq e^{8 \kappa \max \left\{\left|\Gamma_{1}\right|,\left|\Gamma_{2}\right|\right\}}\left|\Phi_{\star}\left(g_{\ell}, g_{\ell-r}\right)\right|$.

It follows that

$\left|\Phi_{\star}(t, s)\right| \leq e^{8 \kappa \max \left\{\left|\Gamma_{1}\right|,\left|\Gamma_{2}\right|\right\}}\left|S_{\kappa}^{r}\right|$.

Since $S_{\kappa}$ is Schur stable, there are $c_{\kappa}>1$ and $d_{\kappa}>0$ such that for all $m \in \mathbb{N},\left|S_{\kappa}^{m}\right| \leq c_{\kappa} e^{-d_{\kappa} m}$. Thus $\left|\Phi_{\star}(t, s)\right| \leq e^{8 \kappa \max \left\{\left|\Gamma_{1}\right|,\left|\Gamma_{2}\right|\right\}} c_{\kappa} e^{-d_{\kappa} r}$. Now, notice that $r \geq \frac{t-s}{4 \kappa}-2$. Consequently,

$\left|\Phi_{\star}(t, s)\right| \leq e^{8 \kappa \max \left\{\left|\Gamma_{1}\right|,\left|\Gamma_{2}\right|\right\}} c_{\kappa} e^{2 d_{\kappa}} e^{-d_{\kappa} \frac{t-s}{4 \kappa}}$.

From (C.1) and (C.2), we deduce that for all $t \geq s$,

$\left|\Phi_{\star}(t, s)\right| \leq e^{8 \kappa \max \left\{\left|\Gamma_{1}\right|,\left|\Gamma_{2}\right|\right\}} c_{\kappa} e^{2 d_{\kappa}} e^{-d_{\kappa} \frac{t-s}{4 \kappa}}$.

This allows us to conclude that (22) is satisfied.

Now, by integrating (21), we obtain that for all $t \geq T$,

$|z(t)|=\left|\Phi_{\star}(t, t-T) z(t-T)+\int_{t-T}^{t} \Phi_{\star}(t, \ell) \varrho(\ell) d \ell\right|$
$\leq p_{1} e^{-p_{2} T}|z(t-T)|+\int_{t-T}^{t} p_{1} e^{-p_{2}(t-\ell)} d \ell \sup _{\ell \in[t-T, t]}|\varrho(\ell)|$

where the last inequality is a consequence of (22).

\section{References}

Blondel, V., \& Tsitsiklis, J. N. (1997). NP-hardness of some linear control design problems. SIAM Journal on Control and Optimization, 35(6), 2118-2127.

Hespanha, J. P., Liberzon, D., Angeli, D., \& Sontag, E. D. (2005). Nonlinear normobservability notions and stability of switched systems. IEEE Transactions on Automatic Control, 50(2), 154-168.

Ji, Z., Feng, G., \& Guo, X. (2007). A constructive approach to reachability realization of discrete-time switched linear systems. Systems \& Control Letters, 56(11), 669-677.

Kim, S., Campbell, S. A., \& Liu, X. (2006). Stability of a class of linear switching systems with time delay. IEEE Transactions on Circuits and Systems - I: Regular Papers, 53(2), 384-393.

Koru, A. T., Delibaşı, A., \& Özbay, H. (2018). Dwell time-based stabilisation of switched delay systems using free-weighting matrices. International Journal of Control, 91(1), 1-11.

Liberzon, D. (2003). Switching in systems and control. Berlin: Birkhäuser. 
Liberzon, D., \& Morse, A. S. (1999). Basic problems in stability and design of switched systems. IEEE Control Systems Magazine, 19(5), 59-70.

Lin, H., \& Antsaklis, P. J. (2009). Stability and stabilizability of switched linear systems: a survey of recent results. IEEE Transactions on Automatic Control, 54(2), $308-322$.

Liu, X., Lin, H., \& Chen, B. M. (2013). Structural controllability of switched linear systems. Automatica, 49(12), 3531-3537.

Mazenc, F., Ahmed, S., \& Özbay, H. (2017). State feedback stabilization of switched systems with delay: trajectory based approach. In Proceedings of the American control conference. Seattle, USA (pp. 4540-4543).

Mazenc, F., \& Malisoff, M. (2015). Trajectory based approach for the stability analysis of nonlinear systems with time delays. IEEE Transactions on Automatic Control, 60(6), 1716-1721.

Mazenc, F., Malisoff, M., \& Niculescu, S.-I. (2017). Stability and control design for time-varying systems with time-varying delays using a trajectory-based approach. SIAM Journal on Control and Optimization, 55(1), 533-556.

Pettersson, S. (2003). Synthesis of switched linear systems. In Proceedings of the 42nd IEEE conference on decision and control. Maui, Hawaii USA (pp. 5283-5288).

Sun, Z., \& Ge, S. S. (2005). Analysis and synthesis of switched linear control systems. Automatica, 41(2), 181-195.

Sun, Z., \& Ge, S. S. (2011). Stability theory of switched dynamical systems. London: Springer.

Sun, Z., Ge, S. S., \& Lee, T. H. (2002). Controllability and reachability criteria for switched linear systems. Automatica, 38(5), 775-786.

Sun, X.-M., Wang, W., Liu, G.-P., \& Zhao, J. (2008). Stability analysis for linear switched systems with time-varying delay. IEEE Transactions on Systems, Man, and Cybernetics - Part B: Cybernatics, 38(2), 528-533.

Tanwani, A., Shim, H., \& Liberzon, D. (2013). Observability for switched linear systems: characterization and observer design. IEEE Transactions on Automatic Control, 58(4), 891-904.

Vu, L., \& Morgansen, K. A. (2010). Stability of time-delay feedback switched linear systems. IEEE Transactions on Automatic Control, 55(10), 2385-2390.

Wu, F., \& Grigoriadis, K. M. (2001). LPV systems with parameter-varying time delays: analysis and control. Automatica, 37(2), 221-229.

Xie, G., \& Wang, L. (2005). Stabilization of switched linear systems with timedelay in detection of switching signal. Journal of Mathematical Analysis and Applications, 305(1), 277-290.

Yan, P., \& Özbay, H. (2005). Robust controller design for AQM and $\mathcal{H}^{\infty}$-performance analysis. In S. Tarbouriech, C. T. Abdallah, \& J. Chiasson (Eds.), Advances in communication control networks (pp. 49-63). Berlin: Springer.

Yan, P., Özbay, H., \& Şansal, M. (2014). Robust stabilization of parameter varying time delay systems by switched controllers. Applied and Computational Mathematics, 13(1), 31-45.

Zhai, G., Hu, B., Yasuda, K., \& Michel, A. N. (2000). Stability analysis of switched systems with stable and unstable subsystems: an average dwell time approach. In Proceedings of the American control conference. Chicago, Illinois (pp. 200-204).

Zhai, G., Hu, B., Yasuda, K., \& Michel, A. N. (2001). Disturbance attenuation properties of time-controlled switched systems. Journal of the Franklin Institute, 338(7), $765-779$.

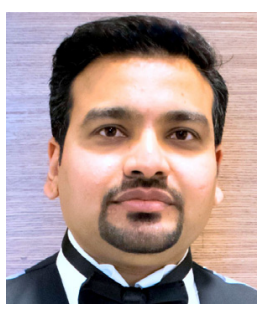

Saeed Ahmed is a Ph.D. candidate at Bilkent University, Ankara, Turkey, under the co-supervision of Hitay Ozbay and Frederic Mazenc. Over the last two years, he was a team member of PHC Bosphore France-Turkey Project between Bilkent University, Ankara, Turkey and INRIA Saclay, France. His current research interests include stability analysis and control of switched and nonlinear systems with time delays, finite-time observer design and output feedback stabilization with limited information, robust and LPV control with an emphasis on biomedical applications.

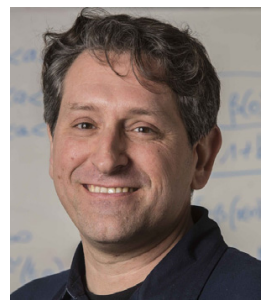

Frédéric Mazenc received his Ph.D. in Automatic Control and Mathematics from the CAS at Ecole des Mines de Paris in 1996. He was a Postdoctoral Fellow at CESAME at the University of Louvain in 1997. From 1998 to 1999, he was a Postdoctoral Fellow at the Centre for Process Systems Engineering at Imperial College. He was a CR at INRIA Lorraine from October 1999 to January 2004. From 2004 to 2009, he was a CR1 at INRIA Sophia-Antipolis. Since 2010, he has been a CR1 at INRIA Saclay. He received a best paper award from the IEEE Transactions on Control Systems Technology at the 2006 IEEE Conference on Decision and Control. His current research interests include nonlinear control theory, differential equations with delay, robust control, and microbial ecology. He has more than 200 peer reviewed publications. Together with Michael Malisoff, he authored a research monograph entitled Constructions of Strict Lyapunov Functions in the Springer Communications and Control Engineering Series.

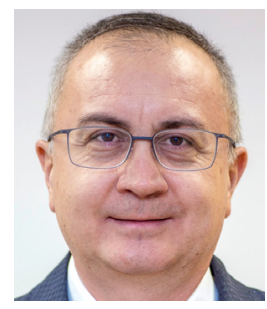

Hitay Özbay is a Professor of Electrical and Electronics Engineering at Bilkent University. He received the B.Sc., M.Eng., and Ph.D. degrees from Middle East Technical University (Ankara, Turkey, 1985), McGill University (Montreal, Canada, 1987), and University of Minnesota, (Minneapolis, USA, 1989), respectively. He was with the University of Rhode Island (1989-1990) and The Ohio State University (1991-2006) where he was a Professor of Electrical and Computer Engineering, prior to joining Bilkent University. He served on the editorial boards of various technical journals, including IEEE Transactions on Automatic Control (1997-1999), SIAM Journal on Control and Optimization (20112014), and Automatica (2001-2007, 2012-present). He is an elected member (for the term 2017-2019) of the Board of Governors of IEEE Control Systems Society, and a general assembly member (since 2013) of the European Control Association (EUCA), representing Turkey. He is a Fellow of IEEE. 